\title{
A secure Certificateless Aggregate Signature Scheme
}

\author{
Baoyuan Kang and Danhui Xu \\ School of Computer science and software \\ Tianjin polytechnic university, Tianjin, 300387, China \\ baoyuankang@aliyun.com, mixueren123123@sina.com
}

\begin{abstract}
Aggregate signatures allow $n$ signatures on $n$ distinct messages from $n$ distinct signers to be aggregated into a single signature that convinces any verifier that $n$ signers do indeed sign the $n$ messages, respectively. The major advantage of utilizing aggregate signatures is to address the security of data and save bandwidth and computations in sensor networks. Recently, people discuss aggregate signature in certificateless public key setting. But some existing certificateless aggregate signature schemes are not secure. In this paper, we analyze the security of Zhang et al. 's certificateless aggregate signature schemes, and propose a new certificateless aggregate signature schemes, and prove the new scheme is existentially unforgeable under adaptive chosen-message attacks under the assumption that computational Diffie-Hellman problem is hard. Furthermore, in signing equation of the proposed scheme user's partial private key and secret value are directly combined with the signed message. So, the scheme is also secure against some inside forgery attack.
\end{abstract}

Keywords: Digital Signature; Aggregate Signature; Certificateless aggregate signature; Security; Bilinear Maps

\section{Introduction}

To surmount the key escrow problem in identity-based public key cryptography, AIRiyami and Paterson originated certificateless public key cryptography [1], there also is a key generation center (KGC) to help users to produce private keys. But, KGC only provides the partial private key. The full private key is generated by the user himself through using the partial private key from KGC and the secret information from himself.

Aggregate signature was first invented by Boneh, Centry, Lynn and Shacham [2]. It combines $n$ signatures from $n$ different signers on $n$ different messages into a single smallsize signature. The single small-size signature is used to examine whether the $n$ signers did sign the $n$ messages, respectively. Aggregate signatures are useful in secure routing [7] and certificate chain compression [2]. The primary advantage of aggregate signature is in saving bandwidth. Such aggregate signature is a good solution for networks of small, battery-powered devices where communication over energy-consuming wireless sensor networks channels [9]. For instance, in unattended wireless sensor network, the lack of real-time communication and resource constraints bring security and performance challenges [19]. To prevent an attacker from altering the data accumulated, forward secure signature [20] is used to sign the accumulated data. But this also causes storage and communication overheads due to the accumulation of the signature of individual data items. Aggregate signature scheme may be developed to address this issue by aggregate the signature of different data items to a single small-size signature.

After the first aggregate signature scheme proposed by Boneh and colleages, a number of aggregate signature schemes were proposed [3, 4, 8, 10, 11, 12, 13]. Recently, people discuss aggregate signature in certificateless public key setting. Some certificateless aggregate signature (CLAS) schemes are proposed [5, 14, 15, 16 ]. Xiong et al. [14] 
contributed a certificateless aggregate signature with constant pairing computations. However, He et al. [6] showed that an adversary in Xiong et al. scheme who knows master key could forge a valid certificateless aggregate signature for any message under any identity. In [16] Zhang et al. proposed a new certificateless aggregate signature scheme. But in the signing equation of the scheme, signer's partial private key and secret value are separated from the signed message. So, Zhang et al.'s scheme is vulnerable to inside attack. The two certificateless aggregate signature schemes [5] proposed by Gong et al. are suffer from same flaws of Zhang et al.'s scheme. Therefore, it is necessary to construct novel certificateless aggregate signature scheme which is provably secure. In this paper, we propose an improved scheme based on Zhang et al.'s scheme [16], and prove that the proposed scheme is existentially unforgeable under adaptive chosenmessage attacks under the assumption of the computational Diffie-Hellman problem being hard. Furthermore, in signing equation of the proposed scheme signer's partial private key and secret value are directly combined with the signed message. So, the proposed scheme is also secure against some potential inside forgery attack. In the era of big data it is vital of data security [21], the secure certificateless aggregate signature (CLAS) schemes are important to dig data.

The rest of the paper is organized as follows. Section 2 introduces cryptographic hardness assumptions and the definition and security model of certificateless aggregate signature. Section 3 reviews Zhang et al.'s certificateless aggregate signature scheme and shows that Zhang et al.'s scheme is vulnerable to inside attack. The improved certificateless aggregate signature scheme is proposed in Section 4. Section 5 discusses the security of improved scheme. Finally, Section 6 concludes this paper.

\section{Preliminaries}

\subsection{Bilinear Maps and Complexity Assumption}

Let $G_{1}$ and $G_{2}$ be additive group and multiplicative group of the same prime $q$ order, respectively. A map $e: G_{1} \times G_{1} \rightarrow G_{2}$ is called a bilinear map if it satisfies the following properties:

(1) Bilinear: $e(a P, b Q)=e(P, Q)^{a b}$ for all $P, Q \in G_{1}, a, b \in Z_{q}^{*}$.

(2) Non-degeneracy: There exists $P, Q \in G_{1}$ such that $e(P, Q) \neq 1$.

(3) Computable: There exists an efficient algorithm to compute $e(P, Q)$ for any

$$
P, Q \in G_{1} \text {. }
$$

Computational Diffie-Hellman (CDH) Problem: Given a generator $P$ of an additive cyclic group $G$ with order $q$, and given $(a P, b P)$ for unknown $a, b \in Z_{q}^{*}$, to compute $a b P$.

\subsection{Definition of Certificateless Aggregate Signature Schemes}

In accordance with [16], A certificateless aggregate signature scheme includes a KGC, an aggregating set $U$ of $n$ users $U_{1}, \cdots, U_{n}$ and an aggregate signature generator. There are six algorithms: Setup, Partial-private-key-extract, userkeygen, sign, aggregate and aggregate verify.

Setup: This algorithm run by KGC, given a security parameter $\tau$, outputs a master key and a list of system parameters params.

Partial-private-key-extract: This algorithm executed by KGC, given a user's identity 
$I D_{i}$, a parameter list params and a master-key, outputs the user's partial private key $D_{i}$.

Userkeygen: An algorithm run by a user, given the user's identity $I D_{i}$, picks a random $x_{i} \in Z_{q}^{*}$ and produces the user's secret value/public key $x_{i} / P_{i}$.

Sign: An algorithm executed by each user $U_{i}$ in an aggregating set $U$. Its inputs are the parameter list params, a message $m_{i} \in\{0,1\}^{*}, U_{i}$ 's identity $I D_{i}$, his public key $P_{i}$ and his signing key $\left(x_{i}, D_{i}\right)$. The output is a signature $\sigma_{i}$ on message $m_{i}$, which is valid under $U_{i}$ 's identity $I D_{i}$ and his public key $P_{i}$.

Aggregate: An algorithm run by the aggregate signature generator, given an aggregate set set $U$ of $n$ users $U_{1}, \cdots, U_{n}$, the identity $I D_{i}$ of each $U_{i}$, the public key $P_{i}$ of $U_{i}$, and a signature $\sigma_{i}$ on a message $m_{i}$ under identity $I D_{i}$ and public key $P_{i}$ for each user $U_{i} \in U$, outputs an aggregate signature $\sigma$ on messages $m_{1}, \cdots, m_{n}$.

Aggregate verify: Given an aggregate set $U$ of $n$ users $U_{1}, \cdots, U_{n}$, the identity $I D_{i}$ of each $U_{i}$, the public key $P_{i}$ of $U_{i}$, an aggregate signature $\sigma$ on messages $m_{1}, \cdots, m_{n}$, the algorithm outputs true if the aggregate signature is valid, or false otherwise.

\subsection{Security Model of Certificateless Aggregate Signature Schemes}

Based on the security model of certificateless signature scheme [1], there are two types adversaries $A_{1}$ and $A_{2}$ for CLAS. Type 1 adversary $A_{1}$ does not have access to the master key, but he can replace the public key of any user. While type 2 adversary $A_{2}$ has access to the master-key but cannot perform public key replacement.

The security of CLAS scheme is modeled in the following two games between a challenger $S$ and an adversary $A_{1}$ or $A_{2}$.

Game 1 (For Type 1 Adversary)

Setup: The challenger $S$ executes this algorithm, given a security parameter $\tau$, the algorithm outputs a master key and the system parameter list params. Then, $S$ sends params to the adversary $A_{1}$ while holds the master key secret.

Attack: The adversary $A_{1}$ executes a polynomial bounded number of queries in an adaptive manner.

Partial-private-key-queries: When $A_{1}$ queries the partial private key of a user with identity $I D_{i}, S$ outputs the partial private key $D_{i}$ for the user.

Public-key queries: When $A_{1}$ queries the public key of whose identity is $I D_{i}, S$ outputs it.

Secret-Value queries: When $A_{1}$ queries the secret value of a user whose identity is $I D_{i}$, $S$ outputs the secret value $x_{i}$ (It outputs $\perp$, if the user's public key has been replaced).

Public-key-replacement queries: For any user with identity $I D_{i}$. $A_{1}$ can select a new public key $P_{i}^{\prime}$ for him and record this replacement.

Sign queries: When $A_{1}$ queries a user's (whose identity is $I D_{i}$ ) signature on a message $m_{i}, S$ generates a valid signature $\sigma_{i}$ on message $m_{i}$ under identity $I D_{i}$ and public key 
$P_{i}$

Forgery: $A_{1}$ outputs a set of $n$ users $U^{*}=\left\{U_{1}^{*}, \cdots, U_{n}^{*}\right\}$ with identities set $L_{I D}^{*}=\left\{I D_{1}^{*}, \cdots, I D_{n}^{*}\right\}$ and corresponding public keys set $L_{P K}^{*}=\left\{P_{1}^{*}, \cdots, P_{n}^{*}\right\}, n$ message set $L_{M}^{*}=\left\{m_{1}{ }^{*}, \cdots, m_{n}^{*}\right\}$, and an aggregate signature $\sigma^{*}$.

$A_{1}$ succeeds if and only if

(1) $\sigma^{*}$ is a valid aggregate signature on message $m_{1}{ }^{*}, \cdots, m_{n}{ }^{*}$ under identities $I D_{1}^{*}, \cdots, I D_{n}^{*}$ and the corresponding public keys $P_{1}^{*}, \cdots, P_{n}^{*}$ chosen by $A_{1}$.

(2) At least one identity, let be $I D_{1}^{*} \in L_{I D}^{*}$, has not been submitted during the partial-private key queries and the signature on $m_{1}{ }^{*}$ under $I D_{1}^{*}$ and the corresponding public key $P_{1}^{*}$ has never been queried during the sign queries. Here $P_{1}^{*}$ denotes the public key of the user $U_{1}^{*}$ whose identity is $I D_{1}^{*}$.

Game 2 (For Type 2 Adversary)

Setup: Given a security parameter $\tau, S$ runs the algorithm to obtain a master-key and the system parameter list params. Then $S$ sends params and the master-key to the adversary $A_{2}$.

Attack: The adversary $A_{2}$ executes a polynomial bounded number of queries in an adaptive manner.

Public-key queries: When $A_{2}$ queries the public key of a user (whose identity is $I D_{i}$ ) of his choice. $S$ outputs the public key $P_{i}$ for this user.

Secret-Value queries: When $A_{2}$ queries a user's (whose identity is $I D_{i}$ ) secret value, $S$ outputs the secret value $x_{i}$ for the user.

Sign queries: When $A_{2}$ queries a user's (whose identity is $I D_{i}$ ) signature on a message $m_{i}, S$ replies with a signature $\sigma_{i}$ on message $m_{i}$ under identity $I D_{i}$ and public key $P_{i}$.

Forgery: $A_{2}$ outputs a set of $n$ users $U^{*}=\left\{U_{1}^{*}, \cdots, U_{n}^{*}\right\}$ with identities set $L_{I D}^{*}=\left\{I D_{1}^{*}, \cdots, I D_{n}^{*}\right\}$, public keys set $L_{P K}^{*}=\left\{P_{1}^{*}, \cdots, P_{n}^{*}\right\}, n$ message set $L_{M}^{*}=\left\{m_{1}^{*}, \cdots, m_{n}^{*}\right\}$, and an aggregate signature $\sigma^{*}$.

$A_{2}$ succeeds, if and only if

(1) $\sigma^{*}$ is a valid aggregate signature on message $m_{1}^{*}, \cdots, m_{n}{ }^{*}$ under identities $I D_{1}{ }^{*}, \cdots, I D_{n}{ }^{*}$ and the corresponding public keys form the set $P_{1}^{*}, \cdots, P_{n}^{*}$ chosen by $A_{2}$.

(2) At least one identity, let be $I D_{1}^{*} \in L_{I D}^{*}$, has not been submitted during the secretvalue queries. The signature on $m_{1}^{*}$ under $I D_{1}^{*}$ and the corresponding public key $P_{1}^{*}$ has never been queried during the sign queries.

Definition A CLAS scheme is existentially unforgeable under adaptive chosenmessage attack if and only if the success probability of any polynomial bounded adversary in any of the above two games is negligible. 


\section{The Security of Zhang et al. Certificateless Aggregate Signature Scheme}

In this section we analyze the security of Zhang et al.'s certificateless aggregate signature scheme. Zhang et al.'s scheme is a typical one that is vulnerable to inside forgery attack.

\subsection{Brief review of Zhang et al.'s Scheme}

In [16], Zhang et al. proposed an efficient certificateless aggregate signature scheme that consists of the following six algorithms.

Setup: Given a security parameter 1 , the KGC chooses a cyclic additive group $G_{1}$ which is generated by $P$ with prime order $q$, chooses a cyclic multiplicative group $G_{2}$ of the same order and a bilinear map $e: G_{1} \times G_{1} \rightarrow G_{2}$. The key generation center (KGC) also chooses a random $\lambda \in Z_{q}^{*}$ as the master-key and sets $P_{T}=\lambda P$, chooses cryptographic hash functions $H_{1}:\{0,1\}^{*} \rightarrow G_{1}, H_{2}:\{0,1\}^{*} \rightarrow G_{1}, H_{3}:\{0,1\}^{*} \rightarrow G_{1}$. The system parameter list is params $=\left(G_{1}, G_{2}, e, P, P_{T}, H_{1}, H_{2}, H_{3}\right)$. The message space is $\mu=\{0,1\}^{*}$.

Partial-Private-Key-Extract: This algorithm accepts params, master-key $\lambda$ and a user's identity $I D_{i} \in\{0,1\}^{*}$. It generates the partial private key for the user as follows:

(1) Computes $Q_{i}=H_{1}\left(I D_{i}\right)$.

(2) Outputs the partial private key $D_{i}=\lambda Q_{i}$.

UserKeyGen: This algorithm takes as input a user's identity $I D_{i}$, selects a random $x_{i} \in Z_{q}^{*}$ and sets his secret value/public key as $x_{i} / P_{i}=x_{i} P$.

Sign: To sign a message $M_{i} \in \mu$ using the signing key $\left(x_{i}, D_{i}\right)$, the signer, whose identity is $I D_{i}$ and the corresponding public key is $P_{i}$, first chooses a state information $\Delta$ (for our scheme, we can choose some elements of the system parameters as $\Delta$ ), then performs the following steps:

(1) Chooses a random $r_{i} \in Z_{q}^{*}$, compute $R_{i}=r_{i} P$.

(2) Computes $W=H_{2}(\Delta), S_{i}=H_{3}\left(\Delta\left\|M_{i}\right\| I D_{i}\left\|P_{i}\right\| R_{i}\right)$.

(3) Computes $V_{i}=D_{i}+x_{i} W+r_{i} S_{i}$.

(4) Outputs $\sigma_{i}=\left(R_{i}, V_{i}\right)$ as the signature on $M_{i}$.

Aggregate: Anyone can act as an aggregate signature generator who can aggregate a collection of individual signatures that use the same state information $\Delta$. For an aggregating set $U$ (which has the same state information $\Delta$ ) of $n$ users $U_{1}, \ldots, U_{n}$ with identities $I D_{1}, \ldots, I D_{n}$ and the corresponding public keys $P_{1}, \ldots, P_{n}$ and message-signature pairs $\left(M_{1}, \sigma_{1}=\left(R_{1}, V_{1}\right)\right), \ldots,\left(M_{n}, \sigma_{n}=\left(R_{n}, V_{n}\right)\right)$ from $U_{1}, \ldots, U_{n}$, respectively, the aggregate signature generator computes $V=\sum_{i=1}^{n} V_{i}$ and outputs the aggregate signature $\sigma=\left(R_{1}, \ldots, R_{n}, V\right)$.

Aggregate Verify: To verify an aggregate signature $\sigma=\left(R_{1}, \ldots, R_{n}, V\right)$ signed by $n$ user 
$U_{1}, \ldots, U_{n}$ with identities $I D_{1}, \ldots, I D_{n}$ and corresponding public keys $P_{1}, \ldots, P_{n}$, on messages $M_{1}, \ldots, M_{n}$ with the same string $\Delta$, the verifier performs the following steps:

(1) Computes $W=H_{2}(\Delta)$, and for all $i, 1 \leq i \leq n$ compute $Q_{i}=H_{1}\left(I D_{i}\right)$, $S_{1}=H_{3}\left(\Delta\left\|M_{i}\right\| I D_{i}\left\|P_{i}\right\| R_{i}\right)$.

(2) Verifies $e(V, P) \stackrel{?}{=} e\left(P_{T}, \sum_{i=1}^{n} Q_{i}\right) e\left(W, \sum_{i=1}^{n} P_{i}\right) \prod_{i=1}^{n} e\left(S_{i}, R_{i}\right)$.

(3) If the equation holds, outputs true. Otherwise, outputs false.

\subsection{Attack on Zhang et al.'s Scheme}

According to the concept of inside attack on aggregate signature proposed in [17, 18], there is an inside attack on Zhang et al.'s scheme.

Let $I D_{1}$ and $I D_{2}$ be identities of two signers $U_{1}, I D_{2}$, respectively. $U_{1}$ and $U_{2}$ declare that they produce a certificateless aggregate signature $\sigma=\left(R_{1}, R_{2}, V\right)$ on messages $\left(M_{1}, M_{2}\right)$ for $\left(I D_{1}, I D_{2}\right)$. In the light of the design of Zhang et al.'s scheme, Of course, $U_{1}$ and $U_{2}$ should sign $M_{1}, M_{2}$, respectively. But, $U_{1}$ and $U_{2}$ may maliciously do as following:

1. $U_{1}$ and $U_{2}$ Choose $r_{1}, r_{2} \in_{R} Z_{q}^{*}$ and compute $R_{1}=r_{1} \cdot P$ and $R_{2}=r_{2} \cdot P$,

2. $U_{1}$ and $U_{2}$ cooperate to compute

$$
\begin{aligned}
& W=H_{2}(\Delta), S_{1}=H_{3}\left(\Delta\left\|M_{1}\right\| I D_{1}\left\|P_{1}\right\| R_{1}\right), S_{2}=H_{3}\left(\Delta\left\|M_{2}\right\| I D_{2}\left\|P_{2}\right\| R_{2}\right) \\
& V_{1}^{*}=D_{1}+x_{1} W+r_{2} S_{2}, V_{2}^{*}=D_{2}+x_{2} W+r_{1} S_{1} .
\end{aligned}
$$

Note they have not signed $M_{1}$ and $M_{2}$, respectively.

3. They declare that they generate certificateless aggregate signature $\sigma=\left(R_{1}, R_{2}, V^{*}\right)$ on messages $\left(M_{1}, M_{2}\right)$ for $\left(I D_{1}, I D_{2}\right)$. Here $V^{*}=V_{1}^{*}+V_{2}^{*}$.

In fact, $V^{*}=V_{1}^{*}+V_{2}^{*}=V_{1}+V_{2}=V$. So, the verification equation

$$
e\left(V^{*}, P\right)=e\left(P_{T}, Q_{1}+Q_{2}\right) e\left(W, P_{1}+P_{2}\right) e\left(S_{1}, R_{1}\right) e\left(S_{2}, R_{2}\right)
$$

holds, $U_{1}$ and $U_{2}$ successfully forge an aggregate signature for $\left(I D_{1}, I D_{2}\right)$ on $\left(M_{1}, M_{2}\right)$. So, Zhang et al.'s scheme is subjected to inside forgery attack.

\section{A New Certificateless Aggregate Signature Scheme}

To overcome the flaw of Zhang et al. scheme [16], in this section we construct a new CLAS scheme which can be regard as an improvement of Zhang et al. scheme. The new scheme consists of six algorithms, Setup, Partial-private-key-extract, UserKeyGen, Sign, Aggregate, Aggregate Verify. KGC performs Setup algorithm and Partial-private-keyextract algorithm to generate the system parameter and user's partial private key. $n$ distinct signers generate $n$ signatures on $n$ distinct messages. On obtaining the $n$ signatures, anyone can act as an aggregate signature generator to aggregate the $n$ individual signatures into a single signature.

Setup: Given a security parameter $\lambda$, the KGC chooses a cyclic additive group $G_{1}$ which is generated by $P$ and a cyclic multiplicative group $G_{2} . G_{1}$ and $G_{2}$ have the same order $q$. Then, KGC chooses a bilinear map $e: G_{1} \times G_{1} \rightarrow G_{2}$ and random $s \in Z_{q}^{*}$ as the master key and sets system public $P_{p u b}=s P$, picks four cryptographic hash functions 


$$
H_{1}:\{0,1\}^{*} \rightarrow G_{1}, H_{2}:\{0,1\}^{*} \rightarrow Z_{q}, H_{3}:\{0,1\}^{*} \rightarrow G_{1}, H_{4}:\{0,1\}^{*} \rightarrow G_{1} .
$$

The system parameter list is params $=\left(G_{1}, G_{2}, e, P, P_{p u b}, H_{1}, H_{2}, H_{3}, H_{4}\right)$. The message space is $\mu=\{0,1\}^{*}$.

Partial-Private-Key-Extract: Given master key $s$ and a user's identity $I D_{i} \in\{0,1\}^{*}$, this algorithm generates the partial private key for the user as follows:

(1) Computes $Q_{i}=H_{1}\left(I D_{i}\right)$.

(2) Outputs the partial private key $D_{i}=s Q_{i}$.

UserKeyGen: Given a user's identity $I D_{i}$, This algorithm picks a random $x_{i} \in Z_{q}^{*}$ as the user's secret value, and computes his public key as $P_{i}=x_{i} P$.

Sign: After choosing a state information $w$, A signer with identity $I D_{i}$ and public key $P_{i}$, signs a message $m_{i} \in \mu$ using the signing key $\left(x_{i}, D_{i}\right)$ by the following steps:

(1) Chooses a random $r_{i} \in Z_{q}^{*}$, computes $R_{i}=r_{i} P$.

(2) Computes $h_{2 i}=H_{2}\left(m_{i}, I D_{i}, P_{i}, R_{i}\right), H_{3 i}=H_{3}\left(m_{i}, I D_{i}, P_{i}, R_{i}\right), T=H_{4}(w)$

(3) Computes $V_{i}=h_{2 i} D_{i}+x_{i} H_{3 i}+r_{i} T$.

(4) Outputs $\sigma_{i}=\left(R_{i}, V_{i}\right)$ as the signature on $m_{i}$.

Aggregate: For the same state information $w$ and an aggregating set $U$ (which has the same state information $w$ ) of $n$ users $U_{1}, \cdots, U_{n}$ with identities $I D_{1}, \cdots, I D_{n}$ and the corresponding public keys $P_{1}, \cdots, P_{n}$ and message-signature pairs $\left(\left(m_{1}, \sigma_{1}=\left(R_{1}, V_{1}\right)\right), \cdots,\left(m_{n}, \sigma_{n}=\left(R_{n}, V_{n}\right)\right)\right.$ from $U_{1}, \cdots, U_{n}$, respectively, any aggregate signature generator can computes $V=\sum_{i=1}^{n} V_{i}$ and outputs the aggregate signature $\sigma=\left(R_{1}, \cdots, R_{n}, V\right)$.

Aggregate Verify: To verify an aggregate signature $\sigma=\left(R_{1}, \cdots, R_{n}, V\right)$ signed by $n$ user $U_{1}, \cdots, U_{n}$ with identities $I D_{1}, \cdots, I D_{n}$ and corresponding public keys $P_{1}, \cdots, P_{n}$, on messages $m_{1}, \cdots, \mathrm{m}_{n}$, the verifier executes the following steps:

(1) Computes $Q_{i}=H_{1}\left(I D_{i}\right), h_{2 i}=H_{2}\left(m_{i}, I D_{i}, P_{i}, R_{i}\right), H_{3 i}=H_{3}\left(m_{i}, I D_{i}, P_{i}, R_{i}\right)$, for all $i, 1 \leq i \leq n$, and $T=H_{4}(w)$.

(2) Verifies $e(V, P) \stackrel{?}{=} e\left(T, \sum_{\mathrm{i}=1}^{n} R_{i}\right) e\left(P_{p u b}, \sum_{i=1}^{n} h_{2 i} Q_{i}\right) \prod_{i=1}^{n} e\left(H_{3 i}, P_{i}\right)$.

(3) If the equation holds, outputs true. Otherwise, outputs false.

\section{Security Proof}

Theorem 1. In the random oracle model, our certificateless aggregate scheme is existentially unforgeable against type 1 adversary under the assumption that the CDH problem in $G_{1}$ is intractable.

Proof. Let $S_{1}$ be a CDH attacker who receives a random instance $(P, a P, b P)$ of the $\mathrm{CDH}$ problem in $G_{1}$. $A_{1}$ a type 1 adversary who interacts with $S_{1}$ as modeled in Game 1 . We show how $S_{1}$ may use $A_{1}$ to solve the $\mathrm{CDH}$ problem, i.e. to compute $a b P$. 
Setup: Firstly, $S_{1}$ sets system public $P_{p u b}=a P$ and selects params $=\left(G_{1}, G_{2}, e, P, P_{p u b}, H_{1}, H_{2}, H_{3}, H_{4}\right)$

Then he sends params to $A_{1}$.

Attack: $A_{1}$ performs following types of queries in an adaptive manner and we consider hash functions $H_{1}, H_{2}, H_{3}$ and $H_{4}$ as random oracles.

$H_{1}$ queries: $S_{1}$ keeps a list $H_{1}^{\text {list }}$ of tuples $\left(I D_{j}, \alpha_{j}, Q_{j}, c_{j}\right)$. On receiving a $H_{1}$ query on $I D_{i}$ the same answer will be given if the query has been recorded on the list $H_{1}^{\text {list }}$. Otherwise, $S_{1}$ selects $\alpha_{i} \in Z_{q}^{*}$ at random and flips a coin $c_{i} \in\{0,1\}$. If $c_{i}=0, S_{1}$ sets $Q_{i}=\alpha_{i} b P$, adds $\left(I D_{i}, \perp, Q_{i}, c_{i}\right)$ to $H_{1}^{\text {list }}$ and returns $Q_{i}$ as answer. Otherwise, sets $Q_{i}=\alpha_{i} P$, adds $\left(I D_{i}, \alpha_{i}, Q_{i}, c_{i}\right)$ to $H_{1}^{\text {list }}$ and returns $Q_{i}$ as answer.

$H_{2}$ queries: $S_{1}$ keeps a list $H_{2}^{\text {list }}$ of tuples $\left(m_{i}, I D_{i}, P_{i}, R_{i}, \beta_{i}\right)$. Whenever $A_{1}$ queries $H_{2}\left(m_{i}, I D_{i}, P_{i}, R_{i}\right)$, the same answer will be given if the query has been recorded on the list $H_{2}^{\text {list }}$. Otherwise, $S_{1}$ selects a random $\beta_{i} \in Z_{q}^{*}$, adds $\left(m_{i}, I D_{i}, P_{i}, R_{i}, \beta_{i}\right)$ to $\mathrm{H}_{2}^{\text {list }}$ and returns $\beta_{i}$ as answer.

$H_{3}$ queries: $S_{1}$ keeps a list $H_{3}^{\text {list }}$ of tuples $\left(m_{i}, I D_{i}, P_{i}, R_{i}, \lambda_{i}, \gamma_{i}\right)$. When $A_{1}$ queries $H_{3}\left(m_{i}, I D_{i}, P_{i}, R_{i}\right)$, the same answer will be given if the query has been recorded on the list $H_{3}^{\text {list }}$. Otherwise, $S_{1}$ picks a random $\gamma_{i} \in Z_{q}^{*}$, computes $\lambda_{i}=\gamma_{i} P$, adds $\left(m_{i}, I D_{i}, P_{i}, R_{i}, \lambda_{i} \cdot \gamma_{i}\right)$ to $H_{3}^{\text {list }}$ and returns $\lambda_{i}$ as answer.

$H_{4}$ queries: $S_{1}$ keeps a list $H_{4}^{\text {list }}$ of tuples $\left(w_{i}, T_{i}, \zeta_{i}\right)$.This list is initially empty. Whenever $A_{1}$ issues a query $H_{4}\left(w_{i}, T_{i}, \zeta_{i}\right)$, the same answer will be given if the query has been recorded on the list $H_{4}^{\text {list }}$. Otherwise, $S_{1}$ selects a random $\zeta_{i} \in Z_{q}^{*}$, computes $T_{i}=\zeta_{i} P$, adds $\left(w_{i}, T_{i}, \zeta_{i}\right)$ to $H_{4}^{\text {list }}$ and returns $T_{i}$ as answer.

Partial-Private-Key queries: $S_{1}$ maintains a list $K^{l i s t}$ of tuples $\left(I D_{j}, x_{j}, D_{j}, P_{i}\right)$. When $A_{1}$ queries a Partial-Private-Key for $I D_{i}$, the same answer from the list $K^{\text {list }}$ will be given if the request has been asked before. Otherwise, $S_{1}$ first does an $H_{1}$ query on $I D_{i}$ and finds the tuple $\left(I D_{j}, \alpha_{j}, Q_{j}, c_{j}\right)$ on $H_{1}^{\text {list }}$, then does as follows:

(1) If $c_{i}=0$, abort.

(2) Else if there's a tuple $\left(I D_{j}, x_{j}, D_{j}, P_{i}\right)$ on $K^{\text {list }}$, set $D_{i}=\alpha_{i} P_{p u b}$, and return $D_{i}$ as answer.

(3) Otherwise, compute $D_{i}=\alpha_{i} P_{p u b}$, set $x_{i}=P_{i}=\perp$, then return $D_{i}$ as answer and add $\left(I D_{j}, x_{j}, D_{j}, P_{i}\right)$ on $K^{\text {list }}$.

Public-Key queries: On receiving a Public-Key query on $I D_{i}$, if the request has been recorded on the list $K^{\text {list }}$, the same answer will be given. Otherwise, $S_{1}$ does as follows:

(1) If there's a tuple $\left(I D_{j}, x_{j}, D_{j}, P_{i}\right)$ on $K^{\text {list }}$ (in this case, the public key $P_{i}$ of $I D_{i}$ is 
$\perp$ ), choose $x_{i}^{\prime} \in Z_{q}^{*}$, compute $P_{i}^{\prime}=x_{i}^{\prime} P$, return $P_{i}^{\prime}$ as answer and update $\left(I D_{j}, x_{j}, D_{j}, P_{i}\right)$ to $\left(I D_{j}, x_{j}^{\prime}, D_{j}, P_{j}^{\prime}\right)$.

(2) Otherwise, choose $x_{i} \in Z_{q}^{*}$, compute $P_{i}=x_{i} P$, return $P_{i}$ as answer, set $D_{i}=\perp$ and add $\left(I D_{i}, x_{i}, D_{i}, P_{i}\right)$ to $K^{\text {list }}$.

Secret-Value queries: On receiving a Secret-Value query on $I D_{i}, S_{1}$ first makes public-key query on $I D_{i}$, then finds the tuple $\left(I D_{i}, x_{i}, D_{i}, P_{i}\right)$ on $K^{\text {list }}$ and returns $x_{i}$ as answer (Note that the value of $x_{i}$ maybe $\perp$ ).

Public-Key-Replacement queries: $A_{1}$ can choose a new public key for the user whose identity is $I D_{i}$. On receiving a Public-Key-Replacement query, $S_{1}$ first finds the tuple $\left(I D_{i}, x_{i}, D_{i}, P_{i}\right)$ on $K^{\text {list }}$ (if such a tuple does not exists on $K^{\text {list }}$ or $P_{i}=\perp, S_{1}$ first makes Public-Key query on $I D_{i}$, then $S_{1}$ updates $P_{i}$ to $P_{i}^{\prime}$.

Sign queries: On receives a Sign query on $m_{i}$ by user with identity $I D_{i} . S_{1}$ first recovers $\left(I D_{i}, \alpha_{i}, Q_{i}, c_{i}\right)$ from $H_{1}^{\text {list }},\left(m_{i}, I D_{i}, P_{i}, R_{i}, \beta_{i}\right)$ from $H_{2}^{\text {list }}$ and then generates the signature as follows:

(1) If $c_{i}=0$, chooses $t_{i}, d_{i} \in Z_{q}^{*}$, sets $R_{i}=t_{i}^{-1}\left(d_{i} P-P_{i}-P_{p u b}\right), H_{3 i}=\beta_{i} Q_{i}$, $T=t_{i} \beta_{i} Q_{i}$ and adds $\left(m_{i}, I D_{i}, P_{i}, R_{i}, \beta_{i}, \beta_{i} Q_{i}\right)$ on $H_{3}^{\text {list }}$, and $\left(w_{i}, T_{i}, t_{i}\right)$ on $H_{4}^{\text {list }}$ computes $V_{i}=d_{i} \beta_{i} Q_{i}$, outputs $\sigma_{i}=\left(R_{i}, V_{i}\right)$. Here $\beta_{i}$ is recovered in $\left(m_{i}, I D_{i}, P_{i}, R_{i}, \beta_{i}\right)$ from $\mathrm{H}_{2}{ }^{\text {list }}$.

(2) If $c_{i}=1$, randomly chooses $R_{i} \in G_{1}$, sets $V_{i}=\alpha_{i} \beta_{i} P_{p u b}+\gamma_{i} P_{i}+\zeta_{i} R_{i}$, outputs $\sigma_{i}=\left(R_{i}, V_{i}\right)$. Here $\gamma_{i}$ is recovered from $\left(m_{i}, I D_{i}, P_{i}, R_{i}, \lambda_{i} \cdot \gamma_{i}\right)$ on $H_{3}^{\text {list }}, \zeta_{i}$ is recovered from $\left(w_{i}, T_{i}, \zeta_{i}\right)$ on $H_{4}^{\text {list }}$

Forgery: Eventually, $A_{1}$ returns a set $U$ of $n$ users, whose identities form the set $L_{I D}^{*}=\left\{I D_{1}^{*}, \cdots, I D_{n}^{*}\right\}$ and the corresponding public keys form the set $L_{P K}^{*}=\left\{P_{1}^{*}, \cdots, P_{n}^{*}\right\}$, $n$ messages form the set $L_{m}^{*}=\left\{m_{1}^{*}, \cdots, m_{n}^{*}\right\}$, a forged aggregate signature $\sigma^{*}=\left\{R_{1}^{*}, \cdots, R_{n}^{*}, V^{*}\right\}$. It is required that there exists $I \in\{1, \cdots, n\}$ such that $A_{1}$ has not asked the partial private key for $I D_{I}$. And $A_{1}$ has not made sign query on $\left(m_{I}^{*}, I D_{I}^{*}, P_{I}^{*}\right)$. Without loss of generality, we let $I=1$. The forged aggregate signature must satisfy

Where

$$
e\left(V^{*}, P\right)=e\left(T^{*}, \sum_{i=1}^{n} R_{i}^{*}\right) e\left(P_{p u b}, \sum_{i=1}^{n} h_{2 i}^{*} Q_{i}^{*}\right) \prod_{i=1}^{n} e\left(H_{3 i}^{*}, P_{i}^{*}\right) .
$$

$Q_{i}^{*}=H_{1}\left(I D_{i}^{*}\right), h_{2 i}^{*}=H_{2}\left(m_{i}^{*}, I D_{i}^{*}, P_{i}^{*}, R_{i}^{*}\right), H_{3 i}^{*}=H_{3}\left(m_{i}^{*}, I D_{i}^{*}, P_{i}^{*}, R_{i}^{*}\right), T^{*}=H_{4}\left(\mathrm{w}^{*}\right)$. $S_{1}$ recovers $\left(I D_{i}^{*}, \alpha_{i}^{*}, Q_{i}^{*}, c_{i}^{*}\right)$ from $H_{1}^{\text {list }},\left(m_{i}^{*}, I D_{i}^{*}, P_{i}^{*}, R_{i}^{*}, \beta_{i}^{*}\right)$ from $H_{2}^{\text {list }}$, $\left(m_{i}^{*}, I D_{i}^{*}, P_{i}^{*}, R_{i}^{*}, \lambda_{i}^{*}, \gamma_{i}^{*}\right)$ from $H_{3}^{\text {list }}$ and $\left(w_{i}^{*}, T_{i}^{*}, \zeta_{i}^{*}\right)$ from $H_{4}^{\text {list }}$ for all $i, 1 \leq i \leq n . S_{1}$ proceeds only if $c_{1}^{*}=0, c_{i}^{*}=1$ for all $i, 2 \leq i \leq n$. Otherwise, $S_{1}$ aborts.

Because the forged certificateless aggregate signature must satisfies

$$
e\left(V^{*}, P\right)=e\left(T^{*}, \sum_{i=1}^{n} R_{i}^{*}\right) e\left(P_{p u b}, \sum_{i=1}^{n} h_{2 i}^{*} Q_{i}^{*}\right) \prod_{i=1}^{n} e\left(H_{3 i}^{*}, P_{i}^{*}\right)
$$

We have that 


$$
e\left(P_{p u b}, h_{21}^{*} Q_{1}^{*}\right)=e\left(V^{*}, P\right)\left(e\left(T^{*}, \sum_{i=1}^{n} R_{i}^{*}\right) e\left(P_{p u b}, \sum_{i=2}^{n} h_{2 i}^{*} Q_{i}^{*}\right) \prod_{i=1}^{n} e\left(h_{3 i}^{*}, P_{i}^{*}\right)\right)^{-1}
$$

And by our setting, $Q_{1}^{*}=\alpha_{1}^{*} b P, h_{21}^{*}=\beta_{1}^{*}, h_{31}^{*}=\gamma_{1}^{*} P, T^{*}=\zeta^{*} P$, and for all $i, 2 \leq i \leq n$, $Q_{i}^{*}=\alpha_{i}^{*} P, h_{2 i}^{*}=\beta_{i}^{*}, h_{3 i}^{*}=\gamma_{i}^{*} P$. So, $S_{1}$ can compute

$$
a b P=\left(\beta_{1}^{*} \alpha_{1}^{*}\right)^{-1}\left(V^{*}-\sum_{i=2}^{n} \beta_{i}^{*} \alpha_{i}^{*} P_{p u b}-\sum_{i=1}^{n}\left(\gamma_{i} P_{i}^{*}+\zeta^{*} R_{i}^{*}\right)\right) .
$$

Theorem 2. In the random oracle model, our certificateless aggregate scheme is existentially unforgeable against type 2 adversary under the assumption that the $\mathrm{CDH}$ problem in $G_{1}$ is intractable.

Proof. Let $S_{2}$ be a CDH attacker who receives a random instance $(P, a P, b P)$ of the $\mathrm{CDH}$ problem in $G_{1}$, and has to compute the value of $a b P . A_{2}$ a type 2 adversary who interacts with $S_{2}$ as modeled in Game 2. We show how $S_{2}$ may use $A_{2}$ to solve the CDH problem, i.e to compute $a b P$.

Setup: Firstly, $S_{2}$ selects a random $\eta \in Z_{q}^{*}$ as the master-key, computes system public key $P_{p u b}=\eta P$. Then selects the system parameters

$$
\text { params }=\left(G_{1}, G_{2}, e, P, P_{p u b}, H_{1}, H_{2}, H_{3}, H_{4}\right) .
$$

Then he sends params and the master key $\eta$ to $A_{2}$. Since $A_{2}$ has access to the masterkey, he can do Partial-Private-key-Extract himself.

Attack: $A_{2}$ can perform the following types of queries in an adaptive manner and we need not model the hash functions $H_{1}$ as a random oracle in this case.

$H_{2}$ queries: $S_{2}$ maintains a list $H_{2}^{\text {list }}$ of tuples $\left(m_{i}, I D_{i}, P_{i}, R_{i}, \beta_{i}\right)$. When $A_{2}$ queries $H_{2}\left(m_{i}, I D_{i}, P_{i}, R_{i}\right)$, the same answer will be given if the query has been recorded on the list $H_{2}^{\text {list }}$. Otherwise, $S_{2}$ selects a random $\beta_{i} \in Z_{q}^{*}$, and adds $\left(m_{i}, I D_{i}, P_{i}, R_{i}, \beta_{i}\right)$ to $H_{2}{ }^{l i s t}$, returns $\beta_{i}$ as answer.

$H_{3}$ queries: $S_{2}$ maintains a list $H_{3}^{\text {list }}$ of tuples $\left(m_{i}, I D_{i}, P_{i}, R_{i}, \lambda_{i}, \gamma_{i}\right)$. When $A_{2}$ queries $H_{3}\left(m_{i}, I D_{i}, P_{i}, R_{i}\right)$, the same answer will be given if the query has been recorded on the list $H_{3}^{\text {list }}$ Otherwise, $S_{2}$ selects a random $\gamma_{i} \in Z_{q}^{*}$, computes $\lambda_{i}=\gamma_{i} a P$, adds $\left(m_{i}, I D_{i}, P_{i}, R_{i}, \lambda_{i} \cdot \gamma_{i}\right)$ to $H_{3}^{\text {list }}$ and returns $\lambda_{i}$ as answer.

$H_{4}$ queries: $S_{2}$ maintains a list $H_{4}^{\text {list }}$ of tuples $\left(w_{i}, T_{i}, \zeta_{i}\right)$. When $A_{2}$ issues a query $H_{4}\left(w_{i}, T_{i}, \zeta_{i}\right)$, the same answer will be given if the query has been recorded on the list $H_{4}{ }^{\text {list }}$. Otherwise, $S_{2}$ selects a random $\zeta_{i} \in Z_{q}^{*}$, computes $T_{i}=\zeta_{i} P$, adds $\left(w_{i}, T_{i}, \zeta_{i}\right)$ to $H_{4}^{\text {list }}$ and returns $T_{i}$ as answer.

Public-Key queries: On receiving a Public-Key query on $I D_{i}$, if the request has been recorded on the list $K^{\text {list }}$, the same answer will be given. Otherwise, $S$ selects $x_{i} \in Z_{q}^{*}$ and flips a coin $c_{i} \in\{0,1\}$. If $c_{i}=0, S$ returns $x_{i} b P$, adds $\left(I D_{i}, P_{i}, c_{i}\right)$ to $K^{\text {list }}$. Otherwise, computes $P_{i}=x_{i} P$, and adds $\left(I D_{i}, x_{i}, P_{i}, c_{i}\right)$ to $K^{\text {list }}$ and returns $P_{i}$ as answer.

Secret-Value queries: On receiving a Secret-Value query on $I D_{i}, S$ first finds the 
tuple $\left(I D_{i}, x_{i}, P_{i}, c_{i}\right)$ on $K^{l i s t}$. If $c_{i}=0, S$ aborts, otherwise, returns $x_{i}$ as answer.

Sign queries: On receives a Sign query on $m_{i}$ by user with identity $I D_{i} . S_{2}$ first recovers $\left(I D_{i}, x_{i}, P_{i}, c_{i}\right)$ on $K^{\text {list }},\left(m_{i}, I D_{i}, P_{i}, R_{i}, \beta_{i}\right)$ from $H_{2}^{\text {list }},\left(m_{i}, I D_{i}, P_{i}, R_{i}, \lambda_{i} \cdot \gamma_{i}\right)$ from $H_{3}^{\text {list }}$, and $\left(w_{i}, T_{i}, \zeta_{i}\right)$ from $H_{4}^{\text {list }}$ then generates the signature as follows:

(1) If $c_{i}=0$, randomly chooses $R_{i} \in G_{1}$, and $d_{i} \in Z_{q}^{*}$, set $H_{3 i}=d_{i} P$, adds $\left(m_{i}, I D_{i}, P_{i}, R_{i}, d_{i}, d_{i} P\right)$ on $H_{3}^{\text {list }}$, and computes $V_{i}=\eta \beta_{i} H_{i}\left(I D_{i}\right)+d_{i} x_{i}(b P)+\zeta_{i} R_{i}$, outputs $\sigma_{i}=\left(R_{i}, V_{i}\right)$.

(2) If $c_{i}=1$, uses the standard sign algorithm to generate $\sigma_{i}=\left(R_{i}, V_{i}\right)$ (because $S$ knows the full signing key of the user whose identity is $\left.I D_{i}\right)$, outputs $\sigma_{i}=\left(R_{i}, V_{i}\right)$.

Forgery: Eventually, $A_{1}$ returns a set $U$ of $n$ users, whose identities form the set $L_{I D}^{*}=\left\{I D_{1}^{*}, \cdots, I D_{n}^{*}\right\}$ and the corresponding public keys form the set $L_{P K}^{*}=\left\{P_{1}^{*}, \cdots, P_{n}^{*}\right\}$, $n$ messages form the set $L_{m}^{*}=\left\{m_{1}^{*}, \cdots, m_{n}^{*}\right\}$, a forged aggregate signature $\sigma^{*}=\left\{R_{1}^{*}, \cdots, R_{n}^{*}, V^{*}\right\}$. It is required that there exists $I \in\{1, \cdots, n\}$ such that $A_{1}$ has not asked the partial private key for $I D_{I}$. And $A_{1}$ has not made sign query on $\left(m_{I}^{*}, I D_{I}^{*}, P_{I}^{*}\right)$. Without loss of generality, we let $I=1$. In addition, the forged aggregate signature must satisfy

Where

$$
e\left(V^{*}, P\right)=e\left(T^{*}, \sum_{i=1}^{n} R_{i}^{*}\right) e\left(P_{p u b}, \sum_{i=1}^{n} h_{2 i}^{*} Q_{i}^{*}\right) \prod_{i=1}^{n} e\left(H_{3 i}^{*}, P_{i}^{*}\right) .
$$

$$
Q_{i}^{*}=H_{1}\left(I D_{1}^{*}\right), h_{2 i}^{*}=H_{2}\left(m_{i}^{*}, I D_{i}^{*}, P_{i}^{*}, R_{i}^{*}\right), H_{3 i}^{*}=H_{3}\left(m_{i}^{*}, I D_{i}^{*}, P_{i}^{*}, R_{i}^{*}\right), T^{*}=H_{4}\left(\mathrm{w}^{*}\right) .
$$

$S_{2}$ recovers $\left(m_{i}^{*}, I D_{i}^{*}, P_{i}^{*}, R_{i}^{*}, \beta_{i}^{*}\right)$ from $H_{2}^{\text {list }},\left(m_{i}^{*}, I D_{i}^{*}, P_{i}^{*}, R_{i}^{*}, \lambda_{i}^{*}, \gamma_{i}^{*}\right)$ from $H_{3}^{\text {list }}$ and $\left(w_{i}, T_{i}, \zeta_{i}\right)$ from $H_{4}^{\text {list }}$ for all $i, 1 \leq i \leq n . S_{2}$ proceeds only if $c_{1}^{*}=0, c_{i}^{*}=1$ for all $i, 2 \leq i \leq n$. Otherwise, $S_{2}$ aborts.

Because the forged certificateless aggregate signature must satisfies

$$
e\left(V^{*}, P\right)=e\left(T^{*}, \sum_{i=1}^{n} R_{i}^{*}\right) e\left(P_{p u b}, \sum_{i=1}^{n} h_{2 i}^{*} Q_{i}^{*}\right) \prod_{i=1}^{n} e\left(H_{3 i}^{*}, P_{i}^{*}\right)
$$

We have that

$$
e\left(h_{31}^{*}, P_{1}^{*}\right)=e\left(V^{*}, P\right)\left(e\left(T^{*}, \sum_{i=1}^{n} R_{i}^{*}\right) e\left(P_{p u b}, \sum_{i=1}^{n} h_{2 i}^{*} Q_{i}^{*}\right) \prod_{i=2}^{n} e\left(h_{3 i}^{*}, P_{i}^{*}\right)\right)^{-1}
$$

And by our setting, $h_{21}^{*}=\beta_{1}^{*}, h_{31}^{*}=\gamma_{1}^{*} a P, P_{1}^{*}=x_{1}^{*} b P, T^{*}=\zeta^{*} P$, and for all $i, 2 \leq i \leq n$, $h_{2 i}^{*}=\beta_{i}^{*}, h_{3 i}^{*}=\gamma_{i}^{*} a P, P_{i}^{*}=x_{i}^{*} P$. Hence, $S_{2}$ can compute

$$
a b P=\left(\gamma_{1}^{*} x_{1}^{*}\right)^{-1}\left(V^{*}-\sum_{i=1}^{n}\left(\eta \beta_{i}^{*} Q_{i}^{*}+\zeta^{*} R_{i}^{*}\right)-\sum_{i=2}^{n} x_{i}^{*} \gamma_{i}^{*}(a P)\right) .
$$

Note Our certificateless aggregate scheme is secure against inside attack. Due to separation of user's partial private key and secret value with messages, Zhang et al.'s scheme cannot withstand inside forgery attack. But, in signing equation of the new scheme, user's partial private key and secret value are directly combined with the signed message. So, the new scheme is secure against inside forgery attack. In sensor networks, where with hostile sensors, signature scheme against inside forgery attack is vital to protect data. 


\section{Conclusion}

In this paper, we analyze the security of Zhang et al.'s certificateless aggregate signature scheme, give an improved certificateless aggregate signature scheme, and prove that the new scheme is existentially unforgeable under adaptive chosen-message attacks assuming the computational Diffie-Hellman problem is hard. Furthermore, in signing equation of the new scheme, user's partial private key and secret value are directly combined with the signed message. So, the new scheme is also secure against some inside forgery attack. The new scheme may have applications where many different certificateless signatures need to be compressed into one single small-size signature.

\section{Acknowledgements}

This work was supported by the Research Programs of Applied Basic and Advanced Technology of Tianjin (No. 15JCYBJC15900).

\section{References}

[1] S. AI-Riyami, K. Paterson, Certificateless public key cryptography. In: ASIACRYPT'03, LNCS 2894, pp. 452-473. Springer, Heidelberg (2003).

[2] D. Boneh, C. Gentry, H. Shacham, B. Lynn, Aggregate and verifiably encrypted signatures from bilinear maps. In: EUROCRPYT'03, LNCS, vol. 2656, pp. 416-432. Springer, Heidelberg (2003).

[3] X. Cheng, J. Liu, X. Wang, Identity-based aggregate and verifiably encrypted signatures from bilinear pairing. In: ICCSA'05, LNCS, vol. 3483, pp. 1046-1054. Springer, Heidelberg (2005).

[4] C. Gentry, Z. Ramzan, Identity-based aggregate signature. In: PKC'06, LNCS, vol. 3958, pp. 257-273. Springer, Heidelberg (2006).

[5] Z. Gong, Y. Long, X. Hong, K. Chen, Two certificateless aggregate signatures from bilinear maps. In: Eighth ACIS international conference on software engineering, artificial intelligence, networking, and parallel/distributed computing'07, pp. 188-193

[6] D. He, M. Tian, A note on 'An efficient certificateless aggregate signature with constant pairing computations'. Cryptology eprint Archive, Available online: http://eprint. iacr.org/2012/445.

[7] S.T. Kent, C. Lynn, J. Mikkelson, K. Seo, Secure border gateway protocol (S-BGP)-Real world performance and deployment issues. NDSS, Internet Society (2000).

[8] S. Lu, R. Ostrovsky, A. Sahai, H. Shacham, B. Waters, Sequential aggregate signatures and multisignatures without random oracles. In: EUROCRPYT'06, LNCS, vol. 4004, pp. 465-485. Springer, Heidelberg (2006).

[9] G. Neven, Efficient sequential aggregate signed date. In: EUROCRPYT'08, LNCS, vol. 4965, pp. 52-69. Springer, Heidelberg (2008).

[10] [10] M. Ruckert, D. Schrode, Aggregate and verifiably encrypted signatures from multilinear maps without random oracles. In: ISA'09, LNCS, vol. 5576, pp. 750-759. Springer, Heidelberg (2009).

[11] R. Sakai, K. Ohgishi, and M. Kasahara, Cryptosystems based on pairing. 2000 Symposium on Cryptography and Information Security, Okinawa, Japan, (2000), pp. 26-28.

[12] Z. Shao, Enhanced aggregate signature from pairings. In: CISC'05, LNCS, vol. 3822, pp. 140-149. Springer, Heidelberg (2005).

[13] K. Shim, An ID-based aggregate signatue scheme with constant pairing computations. The Journal of System and Software, vol. 83 (2010), pp. 1873-1880.

[14] H. Xiong, Z. Guan, Z. Chen, F. Li, An efficient certificateless aggregate signature with constant pairing computations. Information Sciences, vol. 10, (2013), pp. 225-235.

[15] N. Yanai, R. Tso, M. Mambo, E. Okamoto, Certificateless ordered sequential aggregate signature scheme. In: Third international conference on intelligent networking and collaborative systems (2011), pp. 662667

[16] L. Zhang, F. Zhang, A new certificateless aggregate signature scheme. Computer Communication vol. 32 (2009), pp. 1079-1085.

[17] B. Kang, On the security of some aggregate signature schemes. Journal of Applied Mathematics, Vol. 2012, Article ID 416137.

[18] B. Kang, ID-based aggregate signature scheme with constant pairing computations: attack and new construction. Journal of Computational Information Systems, no. 16 (2012), pp. 6611-6618

[19] A. Yavuz, P. Ning, Hash-based sequential aggregate and forward secure signature for unattended wireless sensor networks, 2009 6th Annual International Conference on Mobile and Ubiquitous Systems: Networking and Services, MobiQuitous 2009, 2009, 2009 6th Annual International Conference on Mobile and Ubiquitous Systems: Networking and Services, MobiQuitous (2009).

[20] M. Abdalla, S. Miner, C. Namprempre, Forward-secure threshold signature schemes, in: Topics in 
Cryptology - CT-RSA 2001, LNCS, vol. 2020, Springer, Heidelberg, (2001), pp. 441-456.

[21] Q. Zhang, Z. Chen, A weighted kernel possibilistic means algorithm based on cloud computing for clustering big data. International Journal of Communication Systems, vol. 27, no. 9, (2014), pp. 13781391.

\section{Authors}

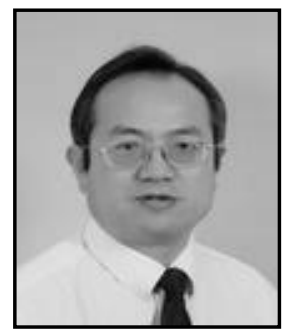

Baoyuan Kang, He received his M.S. in algebra from the shanxi University, and ph.D. in cryptography from Xidian University, People's Republic of China in 1993 and 1999, respectively. From 1993 to 1999, he taught mathematics in Northwestern Polytechnic University. Since 1999 he has taught mathematics and computer science in Central South University. Now he is a professor at Tianjin Polytechnic University. His current research interests are cryptography and information security.

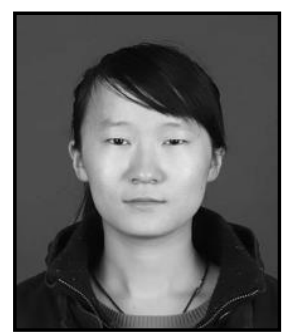

Danhui Xu, She received her B.S. in Computer Science from the Tianjin Polytechnic University University, China in 2013. Now he is a postgraduate student at Tianjin Polytechnic University. His current research interests are cryptography and information security. 
International Journal of Security and Its Applications

Vol. 10, No. 3 (2016) 